\title{
DIAGNÓSTICO EDUCACIONAL EN LA CARRERA DE PEDAGOGÍA EN EDUCACIÓN PARVULARIA
}

\author{
DIAGNÓSTICO EDUCACIONAL NA CARREIRA DE PEDAGOGIA NA EDUCAÇÃO \\ DO JARDIM DE INFÂNCIA
}

\author{
EDUCATIONAL DIAGNOSIS IN THE CAREER OF PEDAGOGY IN KINDERGARTEN \\ EDUCATION
}

\author{
Oscar ROJAS ${ }^{1}$ \\ Amely VIVAS ${ }^{2}$ \\ Doris SOLIS ${ }^{3}$
}

RESUMEN: El presente artículo tiene como objetivo determinar el diagnóstico educacional en la carrera de Pedagogía en Educación Parvularia en la Universidad Miguel de Cervantes ubicada en Santiago de Chile. Para ello se utilizó un enfoque cuantitativo y diseño no experimental transeccional-descriptivo. Los participantes fueron 300 estudiantes que cursaban el primer semestre en la Universidad antes mencionada. Se aplicó muestreo probabilístico intencional y se utilizó cuestionario con escalamiento tipo Likert. Los resultados demostraron que los estudiantes manejan que la UMC no cuenta con la carrera de Educación Parvularia, sin embargo, cuenta con los requerimientos mínimos para dicha creación. Los hallazgos encontrados permitieron determinar que los estudiantes requieren la creación de dicha carrera con el fin de tener procesos establecidos, que permitan la capacitación continua y el aprendizaje permanente, que marca la diferencia con otras instituciones que se han tomado el trabajo de desarrollar esta carrera.

PALABRAS CLAVE: Diagnóstico. Educacional. Carrera. Pedagogía.

RESUMO: Este artigo tem como objetivo determinar o diagnóstico educacional na carreira da Pedagogia da Educação Infantil da Universidade Miguel de Cervantes localizada em Santiago do Chile. Para tanto, utilizou-se uma abordagem quantitativa e um desenho descritivo-transversal não experimental. Os participantes foram 300 alunos que cursavam o primeiro semestre da referida Universidade. Foi aplicada amostra probabilística intencional e questionário do tipo Likert. Os resultados evidenciaram que os alunos percebem que a UMC não possui a carreira de Educação Infantil, no entanto, possui os requisitos mínimos

${ }^{1}$ Universidad Miguel de Cervantes (UMC), Chile. Académico, Dirección de Postgrado e Investigación. Post Doctor en Finanzas, PhD en Economía y Finanzas, Doctorando en Educación, Máster en Educación, MBA, Ingeniero Comercial, Licenciado en Ciencias de la Administración. ORCID: https://orcid.org/0000-0002-67395559. E-mail: oscar.rojas@umcervantesecontinua.cl

${ }^{2}$ Universidad Miguel de Cervantes (UMC), Chile. Académica, Dirección de Postgrado e Investigación. Post Doctor en Estudios Libres, Doctora en Ciencias de la Educación, Maestría en Planificación Educativa, Especialista en Evaluación Educacional, Licenciada en Educación Mención Matemática. ORCID: https://orcid.org/0000-0002-5791-8619.E-mail: amelydvivase@gmail.com

${ }^{3}$ Universidad Miguel de Cervantes (UMC), Chile. Académico. Magíster en Gerencia y Liderazgo en Educación. Licenciada en Educación Integral. Mención: Castellano y Literatura y Ciencias Sociales. ORCID: http://orcid.org/0000-0001-6413-1075. E-mail: dorisolis@hotmail.com

RPGE- Revista on line de Política e Gestão Educacional, Araraquara, v. 25, n. 1, p. 254-266, jan./abr. $2021 . \quad$ e-ISSN:1519-9029 DOI: https://doi.org/10.22633/rpge.v25i1.14728 
para tal criação. Os resultados encontrados permitiram constatar que os alunos requerem a criação da referida carreira para terem processos estabelecidos que permitam a formação contínua e a aprendizagem ao longo da vida, o que faz a diferença com outras instituições que se empenharam no desenvolvimento desta carreira.

PALAVRAS-CHAVE: Diagnóstico. Educacional. Carreira. Pedagogia.

ABSTRACT: This article aims to determine the educational diagnosis in the career of Pedagogy in Early Childhood Education at the Miguel de Cervantes University located in Santiago de Chile. For this, a quantitative approach and a non-experimental transectionaldescriptive design were used. The participants were 300 students who were in the first semester at the aforementioned University. Intentional probability sampling was applied and a Likert-type questionnaire was used. The results showed that the students manage that the UMC does not have the Early Childhood Education career, however, it has the minimum requirements for said creation. The findings found allowed to determine that students require the creation of said career in order to have established processes that allow continuous training and lifelong learning, which makes the difference with other institutions that have taken the work of developing this career.

KEY WORDS: Diagnosis. Educational. Career. Pedagogy.

\section{Introducción}

La educación superior, a nivel mundial, es uno de los pilares principales en los derechos humanos, la libertad, el desarrollo tecnológico, sostenible y la paz; esto debe ser viable para todo individuo a lo largo de la vida; contribuyendo con su formación integral y el mejoramiento continuo de la sociedad. Por lo tanto, la instrucción está dirigida a estudiantes altamente calificados y sujetos responsables en función de atender las necesidades presentes y futuras de la sociedad (LÓPEZ; 2016).

Por lo tanto, la educación es un proceso continuo que acceda al aprendizaje en el desarrollo de competencias, de convivencia, potencialidades, habilidades, destrezas y el afianzamiento de valores individuales y colectivos. Además, la educación superior busca en el desarrollo completo de los ciudadanos en la cual sean capaces de generar cambios y transformaciones en la sociedad presente y futura; haciéndola más equitativa, participativa e inclusiva. La construcción de espacios de aprendizaje continuos que facilitan el pensamiento abierto al mundo (ORGANIZACIÓN DE LAS NACIONES UNIDAS PARA LA EDUCACIÓN, LA CIENCIA Y LA CULTURA, 2013).

En este orden de ideas, en Chile, se ha propuesto mejoras para la formación de profesores y las condiciones laborales en que se desarrolla el ejercicio. Para el 2016 se aprobó 
la Ley 20.903, conocida como la Nueva Política Nacional Docente, donde se aborda la obligatoriedad de realizar una evaluación diagnóstica al ingreso de todas las carreras de pedagogía. La Ley 20.903, que crea el Sistema de Desarrollo Profesional Docente, es uno de los ejes fundamentales de la actual Reforma Educacional. En este sistema, de acuerdo al Ministerio de Educación, se establecen transformaciones para dar solución e intervenir en materias propias de la profesionalidad docente, las necesidades de apoyo a su desempeño y su valoración.

A partir del 2017 entre las medidas que se plantearon en la Ley en el ámbito de la Formación Inicial Docente (en adelante, FID), se exige la acreditación de los programas de pedagogía bajo criterios formativos y de excelencia, tal como lo aborda la Comisión Nacional de Acreditación (CNA) (LEY 20.903 de 2016. Art. 2º, BCN).

Aunado a los nuevos requisitos, se estableció que las y los estudiantes de pedagogía rindan evaluaciones al inicio y al finalizar su carrera. La primera de estas evaluaciones es aplicada por cada una de las universidades y la Ley señala que su propósito es que las universidades cuenten con más información referencial, esperando con ello que se desarrollen acciones de nivelación y acompañamiento, si corresponde (BCN, 2016).

En el caso específico de la Pedagogía Parvularia es determinante en la vida del niño, por cuanto es en esta etapa de su vida donde él toma consciencia, de las cosas que lo rodean y adquiere el dominio de una serie de habilidades que van a configurar su madurez global, tanto intelectual como afectiva. Asimismo, este nivel representa el primer paso del sistema educativo formal, de carácter obligatorio, que tiene como finalidad la formación integral del infante a través de un proceso pedagógico centrado en las necesidades e intereses del niño, desde una perspectiva creativa que garantice la formación de un miembro activo de la sociedad, capaz de proporcionar soluciones dinámicas y efectivas a los diversos requerimientos de la sociedad, lo cual exige al docente, acciones organizadas que conduzcan a facilitar una práctica pedagógica de vanguardia e innovación, dirigida hacia el abordaje de las situaciones problema del proceso de enseñanza y aprendizaje, centrado en su propia creatividad y disposición de enfrentar el quehacer educativo, buscando cada día mayores y mejores recursos.

Esta realidad antes mencionada ha sido motivo de discusión a nivel mundial ante la Declaración de los Derechos del Niño; en el plano académico, las declaraciones sobre Educación Para Todos (Jomtiem), el Proyecto Principal de Educación en América Latina y el Caribe, la Décima Conferencia Iberoamericana de Educación, entre otros, reconocen que lograr una educación de calidad requiere impulsar la educación de la primera infancia, pues 
ella favorece la formación en niveles posteriores, y además se convierte en factor de compensación de desigualdades. Se reafirma entonces, que la educación parvularia es una etapa que sienta las bases para la formación de personalidad, aprendizaje, desarrollo afectivo, capacidad de diálogo y tolerancia en las relaciones interpersonales.

A su vez se adiciona otra situación particular tal como es el déficit de personal docente en Educación Parvularia, donde se aborda que la carencia de docentes formados específicamente para este nivel que exige una atención prioritaria y la baja cobertura de la Educación Parvularia que sólo alcanza en el país para el grado transición el 31\%, distribuidos en un $34 \%$ en la zona urbana y $24 \%$ en la rural, atendiendo solamente a los niños entre 5 y 6 años (VIVAS, 2018).

Teniendo en cuenta estas cifras, se requiere formar nuevos maestros quienes tendrán oportunidad de trabajar en las zonas urbanas y rurales con poblaciones entre 3 y 6 años, partiendo de una nueva lógica de construcción, donde se considere al mismo tiempo el proceso y la calidad de la formación, como los elementos o acciones principales para lograr este doble propósito y que no sea la imposición personalista de un grupo, sino formar al docente en educación parvularia dando respuesta en forma dinámica e integrada a los objetivos de la formación docente, además de orientar la implementación y evaluación del currículo de formación.

Es por ello que, las instituciones de educación superior chilenas tienen su modelo visto como un proyecto educativo, donde se conceptualiza la educación que debe tener contextualizada, en el caso específico la Universidad Miguel de Cervantes (UMC), ubicada en la Zona Metropolitana de Santiago de Chile, es una institución que cuenta con un modelo educativo, desde el año 2010, inspirada en una concepción humanista y cristiana que busca contribuir al bien común de la sociedad, mediante el desarrollo de diversas disciplinas del saber y la formación de profesionales y técnicos, jóvenes, adultos y trabajadores comprometidos con su país, que pertenecen al mundo laboral o que provienen de sectores sociales vulnerables, respondiendo de esta manera a principios de inclusión social.

Por lo tanto, el modelo educativo de la UMC se sustenta en un rol activo-participativo de sus agentes, donde el estudiante es el constructor de un aprendizaje fundamentalmente significativo y autónomo, el docente es un facilitador, guía y tutor de los procesos de enseñanza y aprendizaje y esta construcción del conocimiento está focalizada en la formación profesional e integral donde la Universidad actúa como agente educativo comprometido con el desarrollo de sus estudiantes, generando espacios y dinámicas organizacionales que propician una formación de calidad en diversas modalidades, para aportar al bienestar de las 
personas y de las sociedades. En el modelo educativo, el desarrollo de habilidades es un referente esencial para el proceso de formación del estudiante, se establecen en el perfil de egreso y en la planificación de la enseñanza como un elemento curricular básico y orientador de la formación.

En consecuencia, la UMC plantea un diagnóstico que parte de las causas de lo que se busca mejorar, por ejemplo, los problemas o dificultades de la institución, distinguiendo lo que no funciona como debería, a través del diagnóstico se elige el problema, con sus causas y otros factores que le afecten, sin dejar a un lado que el diagnóstico es un proceso metodológico riguroso y sistemático convirtiéndolo en una actividad científico-profesional.

Partiendo del conocimiento obtenido se toman decisiones educativas para mejorar el aprendizaje y las intervenciones, logrando el apoyo de los procesos de enseñanzaaprendizaje; atención a la diversidad del estudiante; tratamiento de necesidades educativas y asesoramiento tutorial, académico, personal y profesional del educando (GARCÍA, 2011) y contribuyendo al logro de la calidad educativa.

Exaltando esta situación, se aborda que en la UMC donde se forman Licenciados, los docentes pueden tener continuidad de sus estudios a través de la Educación Parvularia, brindando un programa de formación por proyectos lúdico- pedagógicos que integran el saber disciplinar, la construcción de proyectos de aula, la didáctica, la elaboración de materiales educativos y la práctica pedagógica. El desarrollo del proceso formativo, que aportan a la construcción de un modelo pedagógico basado en el aprendizaje colaborativo, socialización y monitoreo.

La relación que debe poseer el programa ajustado a la misión de la UMC se expresa en los valores y tareas compartidas: formar de manera integral ciudadanos libres, de alta calidad profesional, ética y humanística, con capacidad de liderazgo que les permita intervenir de manera decisiva en el mejoramiento de la calidad educativa y de vida de los niños, comprometidos con la consolidación de la democracia, la justicia social, el respeto a los derechos humanos y la paz, para contribuir a la construcción de una sociedad distinta y mejor.

Con relación a la pertinencia social de la educación parvularia, ésta se presenta en los planteamientos de la Carrera de Educación Parvularia teniendo un enfoque humanista, con una visión de desarrollo integral de la niña y el niño. Estos guían al futuro personal docente a hacer su práctica pedagógica tomando en cuenta el contexto sociocultural.

En la práctica no siempre se ejerce la educación parvularia como un aprendizaje para la vida, sino que se visualiza como un aprendizaje para la Escuela. Asimismo, en la formación de las maestras y los maestros persiste el aprendizaje memorístico", con un tipo de 
pensamiento mecanicista, por lo que la educación universitaria tiene el reto de hacer que las y los docentes sean "constructivistas", creativos, para lograr que el niño y la niña aprendan y desarrollen habilidades, destrezas y valores a través del juego.

Otro aspecto que fortalece la pertinencia social de la carrera Pedagogía Parvularia es que se quiere formar un maestro (a) mediador de cultura, que refuerce los valores positivos minimizando los valores destructivos de la sociedad moderna. La UMC tiene el rol fundamental de exaltar los valores predominantes de la sociedad por medio del desarrollo del pensamiento crítico y la formación de docentes en el área de educación parvularia de manera integral, es decir, no debe haber divorcio entre lo que se aprende en el aula (el momento educativo) y la cotidianeidad del niño y la niña.

\section{Formación inicial docente (FID) en Chile}

Actualmente en el país se discute sobre el Proyecto de Ley de Nueva Carrera Docente que ha puesto el énfasis en aspectos como: la formación inicial, la carrera profesional docente, la inducción al ejercicio profesional docente (SÁNCHEZ, 2013) el sistema de remuneraciones y contratación (CRAIG, 2016), o las condiciones para el desempeño docente y la transición misma al nuevo sistema.

En Chile las instituciones de educación superior son las responsables de entregar la Formación Inicial Profesores; el desarrollo de la FID, en cada institución, ha sido de carácter autónomo en relación a las políticas públicas educativas, es decir, las instituciones de FID del país cuentan con cierta libertad curricular para el diseño de sus programas (ÁVALOS, 2014).

De esta manera, hay disciplinas y áreas que aumentan la complejidad de la discusión en el momento de hacer el ejercicio de dar respuesta al porqué existe la necesidad de repensar la formación del profesorado continuamente. Estudiar la formación docente conduce a manejar la competencia profesional docente (PAVIÉ, 2011) así como: la cultura escolar en permanente cambio, la formación inicial docente, la formación continua, la identidad del docente, los perfiles de egreso del profesor y sus respectivas competencias, la inserción en la sociedad de la información, el uso eficiente de las nuevas tecnologías, entre otros.

Aunado a esto, es necesario recalcar que muchos de los problemas que se presentan en la formación profesional del profesor son persistentes en el tiempo y que ya han sido determinados con bastante anterioridad por varios autores: 
ausencia de este cuerpo de conocimientos compartidos -una de las marcas de las profesiones- cuestiona la pertinencia de utilizar el término profesión para la enseñanza. La consideración de la experiencia práctica como la fuente más importante de adquisición de conocimientos y habilidades -situación que configura un saber vulgar técnico, o artesanal pero nunca un saber científico, base del saber profesional- ha conducido durante mucho tiempo a la irrelevancia de la formación para la comunidad académica y los propios profesores (MONTERO, 2001, p. 87).

De acuerdo a lo antes planteado, la formación del docente se debe ser entendida como la formación para la profesión docente.

[...] la preparación y emancipación profesional del docente para elaborar crítica, reflexiva y eficazmente un estilo de enseñanza que promueva un aprendizaje significativo en los alumnos y logre un pensamiento-acción innovador, trabajando en equipo con los colegas para desarrollar un proyecto educativo común (GIMENO, 1982, p. 87).

Este concepto se vincula con los procesos didácticos que posibilitan la preparación, capacitación o perfeccionamiento del docente para su desarrollo o mejora profesional desde el inicio de su carrera. Estos procesos y acciones se desarrollan principalmente en contextos institucionalizados, a los que de modo genérico nos referimos cuando hablamos del ámbito o campo de la formación, o formativo.

Asimismo, la Formación Inicial Docente (FID) es entendida como aquel esquema teórico-práctico que sirve como referencia para la conducción del proceso de formación y es posible ser transferido a otros contextos. Este concepto apela a un esquema que contiene la estructura de los programas, entendida como la organización de distintas áreas que componen el proceso de formación (SÁNCHEZ, 2013). En varios países OCDE la FID considera una relación entre contenido teórico (disciplina), métodos de aprendizaje (conocimiento pedagógico) y experiencia práctica y el desarrollo de habilidades de investigación (PAVIÉ, 2011).

\section{Carrera de Pedagogía}

Chile cuenta con 509 carreras de Pedagogía impartidas en instituciones de educación superior autónomas tal como se observa en el gráfico 1, hasta el 31 de mayo de 2018, un 64\% de ellas se encontraban acreditadas (326 carreras), un 19\% no acreditadas ( 96 carreras) y un 
17\% no se había sometido a procesos de acreditación en el sistema ( 87 carreras). El grueso de la oferta (179 carreras, 35\% del total) se encuentra acreditada en el segundo tramo de acreditación (4 y 5 años); en el primer tramo (2 y 3 años) se encuentra el 16\% de la oferta (79 carreras); y en el tercer tramo de acreditación (6 y 7 años) se contabilizan 68 carreras (13\% de la oferta) (SIES 2007-2019).

Por tipo de institución que imparte las carreras, las universidades pertenecientes al CRUCH tienen el mejor registro de calidad: en promedio, el $74 \%$ de su oferta se encuentra acreditada, un $10 \%$ no acreditada y un $16 \%$ fuera de sistema. En contraste, las universidades privadas poseen un $54 \%$ de su oferta de pedagogía acreditada, un $27 \%$ con decisión de no acreditación y un 19\% fuera de sistema.

Gráfico 1 - Número de carreras de Pedagogía según estado de acreditación.

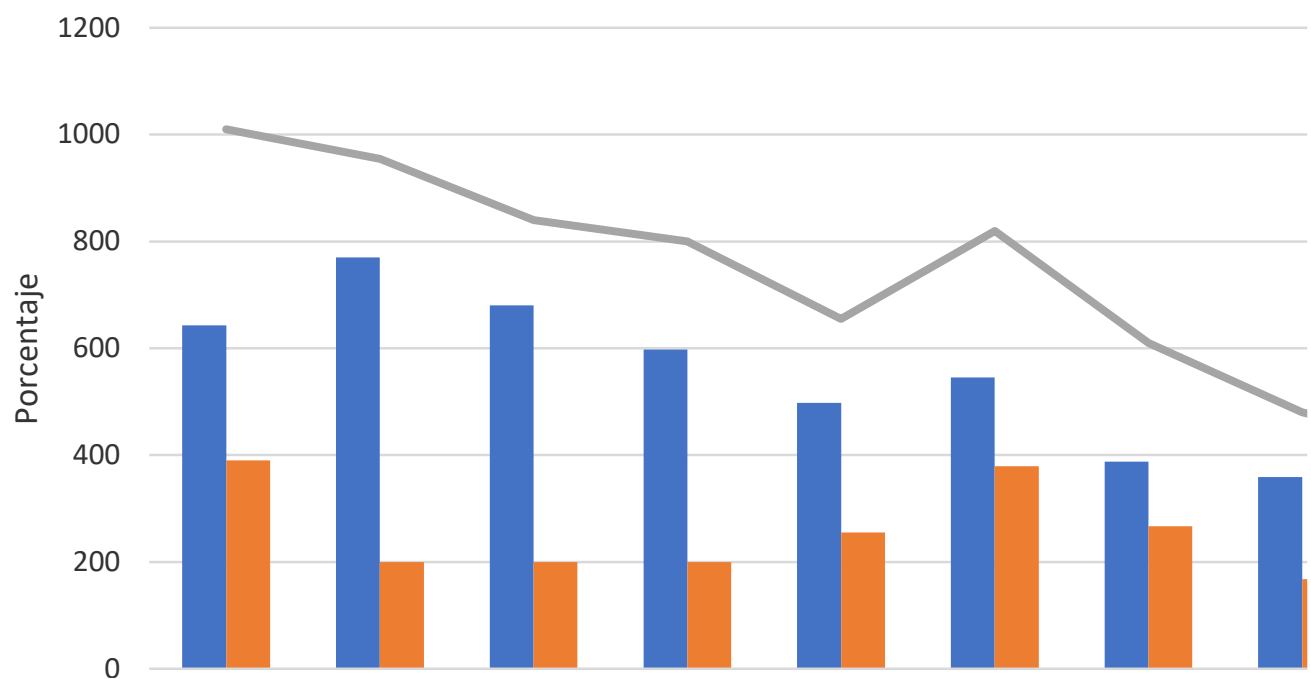

Fuente: SIES 2007-2019

Los nuevos requisitos a regir desde el 2020 implicarían que un 12,6\% de los matriculados en 2019, no podrían haber ingresado a Pedagogía bajo las nuevas condiciones. Esto afecta en particular a las regiones de la zona norte y a las universidades privadas fuera del Consejo de Rectores (Cruch), debido a que una mayor proporción de sus estudiantes no habría cumplido con el estándar 2020 especialmente significativo en las carreras de Educación Parvularia y Educación Diferencial, las que podrían reducir en 23\% y 27\% sus matriculados en 2020 .

Los nuevos requisitos de acceso se basan en puntajes mínimos PSU y notas de enseñanza media. A través del análisis realizado, se determina que el factor de selección más 
relevante sigue siendo la PSU pese a tener sesgos socioeconómicos, ya que el requisito de notas que establece la ley es tan alto, que pocos alumnos logran superarlo. Se presenta para el año 2019, que los 11.844 matriculados que cumplieron con los criterios legales, solo 559 alumnos (5\% de los matriculados) lograron ingresar cumpliendo con el estándar de notas y no de PSU (REYES, 2018).

El docente tiene como tareas básicas la enseñanza y los aprendizajes y, por consiguiente, habrá finalizado cuando el alumnado realmente los haya adquirido (BENEDITO, 1983). Donde el origen de la docencia puede estar relacionado con la aplicación de técnicas de transmisión y métodos de instrucción, además con la relación directa con el estudiante.

\section{Metodología}

Es un estudio descriptivo, en atención a que busca especificar las propiedades importantes de las personas, grupos o cualquier otro fenómeno que sea sometido a análisis y mide o evalúa diversos aspectos, dimensiones o componentes del fenómeno a investigar y su finalidad es analizar la realidad de los hechos, basado en la aplicación, principalmente, de una metodología cuantitativa complementado con un instrumento para la recolección de datos a fin de conocer la complejidad del objeto de estudio.

El estudio fue parte del enfoque cuantitativo del tipo descriptivo y exploratorio en cuanto a su objetivo general (HERNÁNDEZ; FERNÁNDEZ; BAPTISTA, 2014). En cuanto a la población, estuvo constituido por los estudiantes de pregrado de la Licenciatura en Educación de la UMC, es decir, 300 estudiantes. Para la obtención de la muestra se utilizó un muestreo censo poblacional, resaltando que se tomó como muestra la totalidad de la población. La indagación pretendió contribuir con conocimientos científicos válidos para los sujetos objeto de estudio.

El instrumento aplicado fue un cuestionario con escalamiento tipo Likert con tres alternativas de respuestas: De acuerdo (DA, 3), Neutro (N, 2) y En desacuerdo (ED, 1), con un total de quince preguntas. En efecto, se plantearon el desarrollo de dos variables: Diagnóstico institucional y educación parvularia.

Es importante destacar que la validez del instrumento se efectuó a través del análisis de contenido por medio de tres expertos en metodología, estadística y supervisores educativos. Los mismos acordaron que el instrumento se encontraba adecuado de acuerdo a las variables de estudio. Posteriormente, se aplicó una prueba piloto a 60 docentes, vía correo 
electrónico, quienes no pertenecían a la muestra de estudio, pero con características semejantes a la muestra. De esta manera, se usó el coeficiente de alfa de cronbach, obteniendo un valor de 0.97 , considerándose confiable.

En cuanto al procedimiento de recolección de datos, se envió por correo electrónico la invitación a los estudiantes que estudian en pregrado, en la cual se invitaban a participar en el estudio y se les informó acerca de los objetivos y la intención de la misma. Consecutivamente, fue enviado el instrumento y las instrucciones para responder a los ítems formulados. El análisis de los datos se realizó a través del paquete estadístico para las ciencias sociales (SPSS), representándose por medio de tablas en la cual se reflejó las frecuencias absolutas y los porcentajes de las respuestas emitidas por sujetos de estudio.

\section{Resultados}

La descripción de los datos obtenidos por cada variable, se utilizó la distribución de frecuencia absoluta y relativa de cada ítem y como técnica de representación gráfica se empleó el diagrama de barras. Al aplicar la encuesta a los estudiantes de Licenciatura en Educación de la Universidad Miguel de Cervantes Chile, se llegó a los siguientes resultados: Como se puede apreciar en la tabla 1, se muestran las frecuencias absolutas y las frecuencias relativas porcentuales (\%) de cada una de las alternativas de respuestas presentes en la evaluación de los indicadores. A su vez la distribución de los datos se denota por ítem e indicador.

Tabla 1 - Variable: Diagnóstico. Dimensión: Creación de la carrera de pedagogía parvularia

\begin{tabular}{|c|c|c|c|}
\hline ITEMS & $\begin{array}{c}\text { DE ACUERDO } \\
\text { (3) }\end{array}$ & $\begin{array}{c}\text { NEUTRO } \\
\text { (2) }\end{array}$ & EN DESACUERDO (1) \\
\hline $\begin{array}{l}\text { 1. ¿La Universidad Miguel de } \\
\text { Cervantes cuenta con la carrera de } \\
\text { Pedagogía Parvularia? }\end{array}$ & $0 \%$ & $0 \%$ & $100 \%$ \\
\hline $\begin{array}{l}\text { 2. ¿Considera fundamental la creación } \\
\text { de la carrera de pedagogía Parvularia? }\end{array}$ & $100 \%$ & $0 \%$ & $0 \%$ \\
\hline \multicolumn{1}{|c|}{ TOTAL } & $100 \%$ & $0 \%$ & $0 \%$ \\
\hline
\end{tabular}

Fuente: Elaboración propia

Los resultados obtenidos, sin duda ofrecen un catálogo amplio de lecturas, entre ellas, el (100\%) por ciento de la muestra consideran estar en desacuerdo en que la UMC no cuenta con la carrera de Pedagogía Parvularia. Además, el 100\% consideran estar de acuerdo en la creación de la carrera de Pedagogía Parvularia en la Universidad Miguel de Cervantes, Chile. 
Tabla 2 - Variable: Diagnóstico. Indicador: Condiciones en la UMC

\begin{tabular}{|c|c|c|c|}
\hline ITEMS & DE ACUERDO (3) & NEUTRO (2) & $\begin{array}{c}\text { EN } \\
\text { DESACUERDO }\end{array}$ \\
\hline $\begin{array}{l}\text { 3. iExisten leyes o reglamentos } \\
\text { suficientes y adecuados para regir el } \\
\text { proceso de formación de la carrera de } \\
\text { Pedagogía en Parvularia? }\end{array}$ & $100 \%$ & $0 \%$ & $0 \%$ \\
\hline $\begin{array}{l}4 . \quad \text { Cuenta la UMC con la } \\
\text { infraestructura suficiente y adecuada } \\
\text { de manera que se facilite el proceso } \\
\text { de enseñanza-aprendizaje de la } \\
\text { carrera de Pedagogía en Parvularia? }\end{array}$ & $100 \%$ & $0 \%$ & $0 \%$ \\
\hline \multicolumn{1}{|c|}{ TOTAL } & $100 \%$ & $0 \%$ & $0 \%$ \\
\hline
\end{tabular}

Fuente: Elaboración propia

Se observa de manera clara la distribución porcentual (\%) de los resultados que arrojaron los ítems. Conviene mencionar que las alternativas de respuestas disponibles en el instrumento fueron $100 \%$ consideran estar de acuerdo de que existen leyes o reglamentos suficientes y adecuados para regir el proceso de formación de la carrera de pedagogía en parvularia. El ítem 4 fue considerado por los encuestados en un 100\% estar de acuerdo que la UMC cuenta con la infraestructura suficiente y adecuada de manera, que se facilite el proceso de enseñanza-aprendizaje de la carrera de Pedagogía en Parvularia.

En lo referente a las aulas, se califican entre regulares y buenas, condiciones que se modificaran con la readecuación de éstas, debido a remodelaciones surgidas recientemente. Respecto a la biblioteca, se manifiesta que cuenta con las condiciones deseables para un buen funcionamiento.

\section{Discusión}

Los resultados obtenidos, se puede evidenciar que la carrera de pedagogía en educación parvularia, en la cual los estudiantes reconocen la importancia y relevancia de la creación de la carrera de Pedagogía en Educación Parvularia. Esto es bastante significativo, para el presente estudio, porque los estudiantes, consideran un espacio de oportunidad para la carrera que se propone, porque en la UMC se está desarrollando la Licenciatura em Educación, además, permite el seguimiento al magíster en sus dos menciones: Magíster Profesional en Educación Mención Curriculum y Evaluación Basado en Competencias y Magíster Profesional en Educación Mención Gestión de Calidad o puede seguir los cursos de diplomados y postítulos.

RPGE- Revista on line de Política e Gestão Educacional, Araraquara, v. 25, n. 1, p. 254-266, jan./abr. 2021. 
Por otra parte, los datos obtenidos en el indicador Condiciones en la UMC, se observa que la gran mayoría de los estudiantes están de acuerdo en cuanto a que existen leyes entre otros documentos que sustentan la creación de la carrera. La carrera de Educación Parvularia responden a:

Necesidad de preparar a los docentes para el manejo y aprovechamiento de los recursos tecnológicos e informativos que se implementan en el nivel de Parvularia.

Formación profesional en la carrera de Educación Parvularia que tengan a su cargo los niños y niñas, de este nivel, por el manejo de la información dentro de sus instituciones educativas y por la interacción de los elementos humanos de la misma, siendo estos poseedores de conocimientos relevantes (UMC, 2020).

\section{REFERENCIAS}

BENEDITO, L. Diversidad y políticas públicas. Santiago de Chile. Editado por el Instituto de Asuntos Públicos de la Universidad de Chile, 1983.

CHILE. Ministerio de Educación. Ley n. 20.903, 04 de marzo. Disponible: https://www.bcn.cl/leychile/navegar?idNorma=1087343. Acceso: 10 jun. 2019.

GARCÍA, L. Institucionalidad para las políticas de primera infancia y resguardo de la calidad de la educación inicial desde la perspectiva de derechos. Docencia, n. 48, p. 46-58, 2011.

GIMENO, J. La pedagogía por objetivos. Obsesión por la eficiencia. Madrid: Morata, 1982.

HERNÁNDEZ, R.; FERNÁNDEZ, C; BAPTISTA, P. Metodología de la Investigación. Editorial: McGraw Hill. México, 2014.

\section{LÓPEZ, J. La Calidad en Educación Superior y su Relación con el Desarrollo Social}

Inclusivo. 2016. Tesis (Doctoral) - Universidad de Chile, Chile, 2016. Disponible:

http://repositorio.uchile.cl/bitstream/handle/2250/140834/La-calidad-en-Educacion-Superiory-su-relacion-con-el-desarrollo-social inclusivo.pdf?sequence=1\&isAllowed=y. Acceso: 10 jun. 2019.

MONTERO, L. Radiografía de la institucionalidad para la primera infancia en Chile. Serie Desafíos en la educación de primera infancia. UDP, n. 20, p. 1-9, 2011.

ORGANIZACIÓN DE LAS NACIONES UNIDAS PARA LA EDUCACIÓN, LA CIENCIA Y LA CULTURA. Situación Educativa de América Latina y el Caribe. Hacia una educación para todos, 2015. Disponible:

http://www.unesco.org/new/fileadmin/MULTIMEDIA/FIELD/Santiago/pdf/situacioneducativa-mexico-2013.pdf. Acceso: 10 jun. 2019.

PAVIÉ, O. Chilenos Todos? La construcción social de la nación (1810-1840). Santiago, LOM, 2011 
REYES, L. La demanda y oferta de la Educación temprana en Chile. Centro de Estudios Públicos (CEP), v. 126, p. 45-86, 2018.

SIES 2007-2019. Panorama de la Educación Superior en Chile 2014. Chile: División de Educación Superior, Ministerio de Educación, 2014.

VIVAS, A. Educación Preescolar: Evidencia y Desafíos para Chile. Serie Informe Social, Libertad y Desarrollo, v. 138, p. 1-28, 2018.

\section{Cómo referenciar este artículo}

ROJAS, O.; VIVAS, A.; SOLIS, D. Diagnóstico educacional na carreira de pedagogia na educação do jardim de infância. Revista on line de Política e Gestão Educacional, Araraquara, v. 25, n. 1, p. 254-266, jan./abr. 2021. e-ISSN:1519-9029. DOI: https://doi.org/10.22633/rpge.v25i1.14728

Enviado: $10 / 11 / 2020$

Revisiones necesarias: $20 / 11 / 2020$

Aprobado: 20/12/2020

Publicado: 02/01/2021 